Proc. of the 11 Int. School on Theoretical Physics Symmetry and Structural Properties of Condensed Matter, Rzeszów 2014

\title{
Strain Designed Magnetic Properties of III-V Magnetic Semiconductors
}

\author{
S. StagraczyŃski ${ }^{a, *}$, C. JAsiukiewicz ${ }^{b}$, V.K. DugaeV $^{b, c}$ And J. Berakdar $^{a}$ \\ ${ }^{a}$ Institut für Physik, Martin-Luther-Universität Halle-Wittenberg, \\ Karl-Freiherr-von-Fritsch Str. 3, 06120 Halle (Saale), Germany \\ ${ }^{b}$ Department of Physics, Rzeszów University of Technology, al. Powstańców Warszawy 6, 35-959 Rzeszów, Poland \\ ${ }^{c}$ Departamento de Física and CeFEMA, Instituto Superior Técnico, Universidade de Lisboa, \\ av. Rovisco Pais, 1049-001 Lisbon, Portugal
}

\begin{abstract}
We present the theoretical analysis of a possibility of the magnetic anisotropy control using various components of the strain tensor in III-V magnetic semiconductor. We used the Kane model of the valence bands for the numerical simulations of the influence of strain on the $\mathrm{Mn}$ doped GaAs valence band structure. Calculating numerically the energy structure of deformed GaMnAs magnetic semiconductor, we also found the total energy of electron system as a function of orientation of the average magnetization vector. Our calculations show how the direction of the magnetization easy axis can be effectively rotated by using different types of deformation.
\end{abstract}

DOI: 10.12693 /APhysPolA.128.219

PACS: 75.50.Pp, 75.30.Gw, 75.80.+q

\section{Introduction}

Realization of the new ideas in spintronics requires, among others, a possibility of effective control of the magnetization via nonmagnetic parameters [1] like, for example, the electric current or an external electric field. Actually, in the recent literature one can find different methods, which have been proposed to rotate the magnetization vector in ferromagnets and magnetic semiconductors without using magnetic field [2-4]. In particular, it was recently discovered that the strong coupling between magnetic and elastic parameters $[3,5]$ allows for the strain to be a very good tool to change the magnetization direction in (Ga,Mn)As magnetic semiconductor.

The GaAs semiconductor does not exhibit magnetic properties but while doped with the Mn atoms, it gains these properties along with growing of the Mn doping. The Mn dopant atom is an acceptor for GaAs semiconductor introducing a hole in the valence band. Besides, the $\mathrm{Mn}^{2+}$ ion in crystal lattice has a localized magnetic moment $5 \mu_{\mathrm{B}}$. The appearance of bulk magnetization is mostly due to the hole-mediated exchange interaction between the magnetic moments of Mn ions. Correspondingly, the electronic structure of the valence band in GaMnAs magnetic semiconductor is the key element of magnetic coupling, which mostly determines all the parameters related to the magnetic ordering of $\mathrm{Mn}$ ions, including the critical temperature of magnetic phase transition, the magnitude of average magnetization, and the parameters of magnetic anisotropy.

It turns out that the increase of average magnetization

*corresponding author; e-mail: ststag@gmail.com can be reached only up to some level of doping, above which the growing number of $\mathrm{Mn}$ ions in the interstitial positions lessen the effect of Mn doping [6, 7]. Many different factors such as the doping level, concentration of defects, contact of GaMnAs layer to the substrate with a different lattice parameter as well as an external stress can be the origin of possible deformations. Such deformations described by including the strain tensor components in the Hamiltonian of holes in the GaMnAs, can strongly modify its band structure. Correspondingly, it can also affect the coupling between magnetic moments and the magnetic anisotropy parameters of the system [8]. In the next sections we show how the magnetic anisotropy can be controlled by the strain when we change the other parameters of the material such as the hole density.

\section{Model}

In our calculations we use the six-level Kane model [9] with effective Hamiltonian containing the standard $k \cdot p$ Luttinger-Kohn part [10] for the valence band with spinorbit interaction [11], the Bir-Pikus part [12] describing the influence of a strain on the band structure, and a magnetic part for the contribution of $p-d$ exchange interaction between the holes and the magnetic moment [13]:

$$
\widehat{\mathcal{H}}=\sum_{\boldsymbol{k}} \psi_{\boldsymbol{k}}^{\dagger}\left(\widehat{H}_{0}+\widehat{H}_{\varepsilon}+\widehat{H}_{m}\right) \psi_{\boldsymbol{k}},
$$

where $\widehat{H}_{0}, \widehat{H}_{\varepsilon}$ and $\widehat{H}_{m}$ are for $k \cdot p$ Hamiltonian of holes in GaAs, strain and magnetic parts, respectively.

In this model four $\Gamma_{8}$ and and two $\Gamma_{7}$ bands are taken explicitly into account, whereas the effects of other energy bands are included by using the second-order perturbation theory. The $\widehat{H}_{\varepsilon}$ term in Eq. (1) includes the components of strain tensor $u_{i j}$, while the $\widehat{H}_{m}$ is a function 
of both, the direction of magnetization $\boldsymbol{M}$ and the $p-d$ exchange parameters, which after Dietl [13] are denoted by $B_{G}$. By changing these parameters one can study the dependence of the band structure on the strain as well as on the magnetic parameters.

\section{Hole concentration}

The hole concentration calculated at a given location of the chemical potential $\mu$ is depending on all the magnetic and strain parameters, which are included in $\widehat{H}_{\varepsilon}$ and $\widehat{H}_{m}$ parts of the Hamiltonian, respectively. It accounts for influence of the strain components, magnetization direction and Mn dopant concentration determining the average magnetization value. The corresponding relation between the total hole concentration $p$ and chemical potential $\mu$ at zero temperature is

$$
p(\mu)=\sum_{n} \int_{\mathrm{BZ}} \frac{\mathrm{d}^{3} \boldsymbol{k}}{(2 \pi)^{3}} \theta\left[E_{n}(\boldsymbol{k})-\mu\right],
$$

where $E_{n}(\boldsymbol{k})$ is the electron energy dispersion in the $n$ th valence energy band determined by the Hamiltonian (1), $\theta(x)$ is the Heaviside step function, and BZ denotes the integration over total first Brillouin zone. Here the Heaviside function is limiting integration by the states near the edge of the valence band, where the $k \cdot p$ approximation is still valid.

We calculated the energy dispersion $E_{n}(\boldsymbol{k})$ in Eq. (2) numerically by diagonalizing the Hamiltonian (1) and then summing up the contributions of all six energy bands. Note that in general case of $M \neq 0$ and $u_{i j} \neq 0$ there is no degeneration of the energy bands in any points of the inverse space, so that the possible band crossing is always accompanied by corresponding splitting of the energy terms near the point of possible crossing [14].

\section{Total energy}

The total energy of the electronic system corresponding to the states filled with electrons, can be calculated as

$$
\mathcal{E}(\mu)=\sum_{n} \int_{\mathrm{BZ}} \frac{\mathrm{d}^{3} \boldsymbol{k}}{(2 \pi)^{3}} E_{n}(\boldsymbol{k})\left\{1-\theta\left[E_{n}(\boldsymbol{k})-\mu\right]\right\} .
$$

Now we can introduce the variation of the total energy of electronic system related to nonzero magnetization and strain

$$
\Delta \mathcal{E}=\mathcal{E}(\mu)-\mathcal{E}_{0}\left(\mu_{0}\right),
$$

where $\mathcal{E}_{0}$ is the energy of electronic system without strain and magnetization while $\mu$ and $\mu_{0}$ are the chemical potentials of both systems corresponding to the same value of hole concentration $p$.

Even though the integral (3) runs over the states where the $k \cdot p$ approximation is not valid anymore, the corresponding energy difference of Eq. (4) does not include contribution of the electronic states with large value of momentum $k$. This justifies the use of $k \cdot p$ approximation for the calculation of energy structure in the strained GaMnAs magnetic semiconductor.

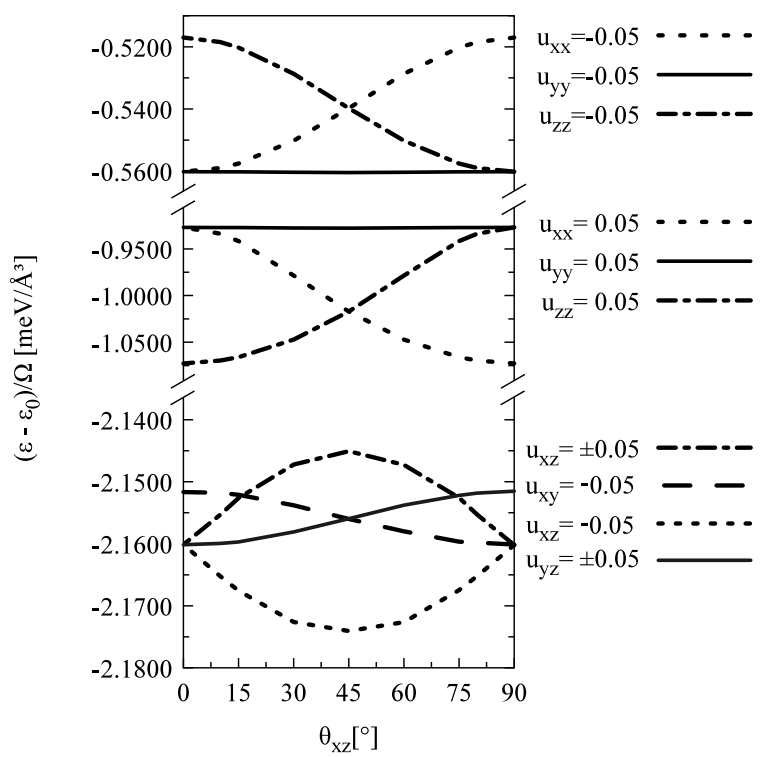

Fig. 1. Variation of the total energy of electronic system with the orientation of magnetization vector $M$ in $z x$ plane for different choice of the strain components $u_{i j}$, fixed hole density $p=4 \times 10^{19} \mathrm{~cm}^{-3}$, and certain value of the magnetization parameter $B_{G}=25 \mathrm{meV}$. The magnetization angles $\theta_{x z}=0^{\circ}$ and $\theta_{x z}=90^{\circ}$ correspond to $\boldsymbol{M}$ parallel to axes $z$ and $x$, respectively.

\section{Results}

In order to study the influence of a strain on the total energy variation (4) we choose the strain tensor $u_{i j}$ with only one component nonequal to zero and changed this value in range of a few percents. In the numerical calculations, we vary the magnetization direction whereas the magnetization strength is controlled by the $B_{G}$ parameter.

Here we assume that the hole density is a constant determined by the doping. Since $\mathrm{Mn}$ is an acceptor in $\mathrm{GaAs}$, and the concentration of $\mathrm{Mn}$ atoms is large, one can assume that the hole density is mostly determined by the Mn doping. By using Eq. (2) we calculated the chemical potential $\mu$ for constant value of $p$, as a function of magnetization orientation for different choice of components of the strain tensor. Then using these results and Eqs. (3) and (4) we calculated the variation $\Delta \mathcal{E}$ of the total energy of electronic system.

The calculations were performed with the parameters of GaAs doped with Mn. Figure 1 shows the results of our calculations. The energy of the electronic system without strain is weakly dependent on the magnetization direction while in the presence of strain the amplitude of total energy variation can be significantly increased.

As shown in Fig. 1, the compression strain along a fourfold axis decreases the energy if the magnetization vector $M$ is directed along this axis, and for the tensile strain the system exhibits the energy minimum for the perpendicular to this axis direction of $\boldsymbol{M}$. Quite different behavior can be observed when the shear strain is applied. 
In that case the compressive/tensile strain places the energy minimum/maximum in the direction corresponding to the angle $\theta_{x z}=45^{\circ}$. The strain perpendicular to the magnetization direction seems not to exhibit the angular dependence of the energy of the system.

Our calculations show similar behavior of the system in a wide range of the strain magnitude and the magnetization value.

\section{Conclusions}

In this paper we demonstrated the possibility of very effective control of the magnetic anisotropy by applying strain to GaMnAs magnetic semiconductor. The magnetization energy minimum along the fourfold axis can be obtained with a compressive strain along this direction. The shear strain $u_{x z}$ can create the energy minimum or an energy barrier between the $\hat{x}$ and $\hat{z}$ directions.

In our calculations we assumed that the variation of strain does not affect the coupling between Mn atoms. Generally, the variation of the position of magnetic moments can affect the magnetic coupling (see, e.g., Ref. [15]). Thus, in these calulations we take the parameter $B_{G}$ constant. The possible justification of this assumption is that the magnitude of average deformation determined by the strain tensor components is about the same for our choice of the deformation type.

\section{Acknowledgments}

This work is supported by the National Science Center in Poland under project of international cooperation "Harmonia" No. DEC-2012/06/M/ST3/00042.

\section{References}

[1] D. Chiba, M. Yamanouchi, F. Matsukura, H. Ohno, Science 301, 943 (2003).

[2] H. Ohno, D. Chiba, F. Matsukura, T. Omiya, E. Abe, T. Dietl, Y. Ohno, K. Ohtani, Nature 408, 944 (2000).
[3] J. Wunderlich, A.C. Irvine, J. Zemen, V. Holy, A.W. Rushforth, E. De Ranieri, U. Rana, K. Výborný, J. Sinova, C.T. Foxon, R.P. Campion, D.A. Williams, B.L. Gallagher, T. Jungwirth, Phys. Rev. B 76, 054424 (2007).

[4] G.V. Astakhov, A.V. Kimel, G.M. Schott, A.A. Tsvetkov, A. Kirilyuk, D.R. Yakovlev, G. Karczewski, W. Ossau, G. Schmidt, L.W. Molenkamp, Th. Rasing, Appl. Phys. Lett. 86, 152506 (2005).

[5] J. Wenisch, C. Gould, L. Ebel, J. Storz, K. Pappert, M.J. Schmidt, C. Kumpf, G. Schmidt, K. Brunner, L.W. Molenkamp, Phys. Rev. Lett. 99, 077201 (2007).

[6] M. Kobayashi, I. Muneta, Y. Takeda, Y. Harada, A. Fujimori, J. Krempaský, T. Schmitt, S. Ohya, M. Tanaka, M. Oshima, V.N. Strocov, Phys. Rev. B 89, 205204 (2014)

[7] T. Jungwirth, K.Y. Wang, J. Mašek, K.W. Edmonds, J. König, J. Sinova, M. Polini, N.A. Goncharuk, A.H. MacDonald, M. Sawicki, A.W. Rushforth, R.P. Campion, L.X. Zhao, C.T. Foxon, B.L. Gallagher, Phys. Rev. B 72, 165204 (2005).

[8] C.S. King, J. Zemen, K. Olejník, L. Horák, J.A. Haigh, V. Novák, A. Irvine, J. Kučera, V. Holý, R.P. Campion, B.L. Gallagher, T. Jungwirth, Phys. Rev. B 2011, 115312 (2011).

[9] E.O. Kane, Phys. Rev. 178, 1368 (1968).

[10] S.L. Chuang, Physics of Optoelectronic Devices, Wiley, New York 1995.

[11] R. Winkler, Spin-Orbit Coupling Effects in TwoDimensional Electron and Hole Systems, Springer, Berlin 2003.

[12] G.L. Bir, G.E. Pikus, Symmetry and Strain-Induced Effects in Semiconductors, Wiley, New York 1974.

[13] T. Dietl, H. Ohno, F. Matsukura, Phys. Rev. B 63, 195205 (2001).

[14] L.D. Landau, E.M. Lifshitz, Quantum Mechanics, Pergamon Press, New York 1977, Ch. XI.

[15] C. Jia, J. Berakdar, EuroPhys. Lett. 85, 57004 (2009). 